\title{
BIORTHONORMAL FILTER BANKS: SOME NECESSARY CONDITIONS, AND ORTHONORMALIZATION
}

\author{
Igor Djokovic and P. P. Vaidyanathan \\ Department of Electrical Engineering \\ California Institute of Technology, Pasadena, CA 91125
}

\begin{abstract}
We first study some properties of nonuniform biorthonormal filter banks. We show that biorthonormality imposes a number of restrictions on the decimation ratios, e.g., they cannot be relatively prime. Also biorthonormality implies orthonormality when all the impulse responses have the same energy. After a brief study of these properties we show how to orthonormalize a nonuniform biorthonormal system by a procedure that is somewhat similar in spirit to (but fundamentally different from) the Gram Schmidt procedure. We then modify the procedure for the purpose of designing biorthonormal filter banks that completely decorrelate the subband signals for a given input statistics.
\end{abstract}

\section{INTRODUCTION}

Fig. 1.1 shows an $M$ channel filter bank, where the decimation ratios $n_{k}$ are integers. We say that the system has the perfect reconstruction (PR) property if $\widehat{x}(n)=x(n)$. (Ignore the subband quantizers for now.) We consider only maximally decimated systems, i.e., $\sum_{k}\left(1 / n_{k}\right)=1$. Let $h_{k}(n)$ and $f_{k}(n)$ denote the impulse responses of the analysis and synthesis filters $H_{k}(z)$ and $F_{k}(z)$ respectively. The PR property means

$$
x(n)=\sum_{i=0}^{M-1} \sum_{k=-\infty}^{\infty} x_{i}(k) f_{i}\left(n-n_{i} k\right) .
$$

For mathematical purposes we will assume that $x(n)$ belongs to the set of finite energy signals ( $l_{2}$ class). We say that the doubly indexed set of functions

$$
\left\{f_{i}\left(n-n_{i} k\right)\right\}
$$

$0 \leq i \leq M-1,-\infty \leq k \leq \infty$ forms a filter-bank like family of functions. These are $n_{i}$-shifted versions of the filter impulse responses $f_{i}(n)$. Note that the space has infinite dimension.

The system is said to be biorthonormal if

$$
\sum_{n} h_{i}\left(n-m n_{i}\right) f_{l}\left(-n+k n_{l}\right)=\delta(i-l) \delta(m-k)
$$

Work supported in parts by National Science Foundation grant MIP 8919196 and funds from Rockwell Inc., and Tektronix, Inc. 0-7803-1254-6/93\$03.00 @ 1993 IEEE for $0 \leq i, l \leq M-1$ and all integers $m, k$. It can be shown that the $P \bar{R}$ property is equivalent to biorthonormailty $[1,2]$; this is fairly subtle, holding only for the maximally decimated system. For example, the two channel system $H_{0}(z)=1+z^{-1}, H_{1}(z)=1-z^{-1}, F_{0}(z)=F_{1}(z)=0.5$ has perfect reconstruction with $n_{0}=n_{1}=1$ (undecimated filter bank), but it does not satisfy biorthonormality!

Biorthonormality can also be expressed as $[1,3]$

$$
\sum_{n} h_{i}(n) f_{l}\left(m g_{i l}-n\right)=\delta(i-l) \delta(m)
$$

where $g_{i l}=\operatorname{gcd}\left(n_{i}, n_{l}\right)$. Equivalently

$$
\left.\left(H_{i}(z) F_{l}(z)\right)\right\rfloor_{g_{i l}}=\delta(i-l) \quad \text { (biorthonormality) }
$$

where $(S(z)) \downarrow_{L}$ represents the $z$ transform of the $L$-fold decimated version of $s(n)$. For the special case of orthonormal filter banks, the perfect reconstruction property implies $F_{k}(z)=\widetilde{H}_{k}(z)$ (where $\widetilde{H}(z) \triangleq H^{*}\left(1 / z^{*}\right)$ ) and the above reduces to

$$
\begin{aligned}
& \left(H_{i}(z) \widetilde{H}_{l}(z)\right) \downarrow_{g_{i l}}=\delta(i-l) \quad \text { (orthonormality) (1.6) } \\
& \text { or, equivalently }\left(F_{i}(z) \widetilde{F}_{l}(z)\right) \downarrow_{g_{i l}}=\delta(i-l) \text {. For the spe- }
\end{aligned}
$$
cial case of uniform filter banks $\left(n_{k}=M\right.$ for all $k$ ) the orthonormal property is equivalent to the paraunitary property of the polyphase matrix [4]. These filter banks have several advantages, documented elsewhere [4].

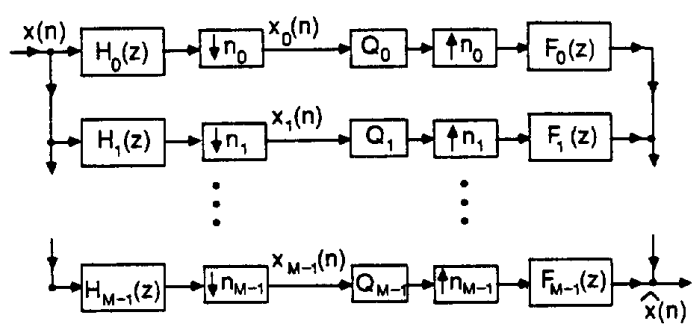

Fig. 1.1. The M-channel filter bank. 
In the uniform case, the existence of PR systems is trivially assured (e.g., the delay chain system [4]), but in the nonuniform case, there exist combinations of integers $\left\{n_{k}\right\}$ such that we can never obtain PR with practical transfer functions [5]. (The existence of ideal, complex coefficient filters giving rise to PR can be easily verified). Nonrational decimators [6],[7] and block decimators [7] are not considered here.

Paper outline. In this paper we first consider some consequences of biorthonormality, and derive some necessary conditions (on $n_{k}$ ) for PR in a maximally decimated system. We then show that whenever $\left\{n_{k}\right\}$ are such that there exists a biorthonormal (i.e., PR) filter bank, then there also exists an orthonormal filter bank. The proof is constructive, somewhat reminiscent of the Gram-Schmidt procedure, but has two fundamental differences. First, the procedure converges after finite number of steps even though the dimension of the space is infinite. Second, the resulting basis continues to have the filter bank like form (1.2). We will then apply the orthogonalization technique for the decorrelation of subband signals of a nonuniform filter bank with rational filters, a result only partially achieved by the Karhunen-Loeve transform (KLT) for the uniform case.

\section{IMPLICATIONS OF BIORTHONORMALITY}

Biorthonormality and perfect reconstruction (PR) property are equivalent in a maximally decimated system [1], though this is not necessarily true in general (See Sec. 1). This equivalence will now be used to derive a number of conclusions. While these pertain only to the maximally decimated case, the system is in general nonuniform (i.e., $n_{i}$ 's are not equal).

1. If any two decimators $n_{i}$ and $n_{l}$ are relatively prime (e.g., $n_{i}=2, n_{l}=3$ ) then it is not possible to achieve PR with rational transfer functions. To see this note that in this case $g_{i l}=1$ and biorthonormality implies $H_{i}(z) F_{l}(z) \stackrel{0}{=}$ which can only be achieved with ideal filters.

2. Suppose all the analysis and synthesis filters have unit energy, i.e., $\sum_{n}\left|h_{i}(n)\right|^{2}=\sum_{n}\left|f_{i}(n)\right|^{2}=1$ for all $i$. Now, biorthonormality (1.4) implies, in particular, that $\sum_{n} h_{i}(n) f_{i}(-n)=1$. By using CauchySchwartz inequality it can be shown that the unit energy property implies $h_{i}(n)=e^{j \theta_{i}} f_{i}^{*}(-n)$. A perfect reconstruction systems with filters so related is necessarily orthonormal [4]. Thus the PR property and unity energy property together imply orthonormality. This does not, however, mean that we can obtain an orthonormal system from a biorthonormal one simply by dividing each transfer function by a constant $[8]$.

3. The PR property implies that $H_{i}(z) F_{i}(z)$ is an $n_{i}$ th band filter [8] (i.e., a Nyquist $\left(n_{i}\right)$ filter [4]). In other words, the impulse response $g(n)$ of this product satisfies $g\left(n_{i} n\right)=0$, for all $n \neq 0$. This can be proved by setting $i=l$ in the biorthonormality relation (1.5). This is a generalization of the fact that for an orthonormal filter bank the filter $H_{i}(z)$ is a spectral factor of a Nyquist $\left(n_{i}\right)$ filter.

4. The PR property implies $\sum_{i} H_{i}(z) F_{i}(z) / n_{i}=1$ [1]. This is a generalization of the power complementary property which is valid only for or thonormal filter banks [4].

5. Assuming the PR property (i.e., biorthonormality), it can be shown [8] that the functions $\left\{f_{i}\left(n-n_{i} k\right)\right\}$ are linearly independent, and form a Riesz basis (unconditional basis) for the $l_{2}$ space.

\section{ORTHONORMALIZATION}

Suppose the decimation ratios $\left\{n_{i}\right\}$ of a (nonuniform) maximally decimated system are such that the PR property (biorthonormality) is possible with rational transfer functions. We show how to derive an orthonormal filter bank (with rational transfer functions) for this set $\left\{n_{i}\right\}$. The process involves two steps, namely orthogonalization, and normalization. We first deal with the latter.

\subsection{Normalization}

Eqn. (1.6) with $i=l$ is called the normalization condition. Thus, the $k$ th analysis or synthesis filter $G_{k}(z)$ is normalized if $\widetilde{G}_{k}(z) G_{k}(z)$ is a $\operatorname{Nyquist}\left(n_{k}\right)$ filter. Given two filters $G_{k}(z)$ and $G_{i}(z)$ satisfying the biorthogonality condition, we show how to modify them so that they are normalized without violating mutual orthogonality. Starting from $G_{k}(z)$ suppose we define

$$
A_{k}(z)=\alpha_{k}\left(z^{n_{k}}\right) G_{k}(z)
$$

It is readily verified that if we choose $\alpha_{k}(z)$ such that

$$
\tilde{\alpha}_{k}(z) \alpha_{k}(z)=\frac{1}{\left.\left(G_{k}(z) \widetilde{G}_{k}(z)\right)\right\rfloor_{n_{k}}}
$$

then $A_{k}(z)$ is normalized, that is

$$
\left.\left(A_{k}(z) \tilde{A}_{k}(z)\right)\right\rfloor_{n_{k}}=1 .
$$

(A spectral factor of the right side of (3.2) can be shown to exist.) Similarly, suppose we construct $A_{i}(z)$ from $G_{i}(z)$. Then $A_{k}(z)$ and $A_{i}(z)$ continue to be orthogonal [8] so that they are in fact orthonormal. Preservation of stability during normalization is discussed in the end.

\subsection{Orthogonalization}

Let $\left\{F_{k}(z)\right\}$ be the set of rational synthesis filters for a maximally decimated PR (biorthonormal) filter bank with decimation ratios $\left\{n_{k}\right\}$. We now describe a procedure to get a new set of rational transfer functions $\left\{G_{k}(z)\right\}$ which are mutually orthogonal, i.e.,

$$
\left.\left(G_{k}(z) \widetilde{G}_{l}(z)\right)\right|_{g_{k l}}=0, \quad k \neq l .
$$


Set $G_{0}(z)=F_{0}(z)$ and let

$$
G_{1}(z)=F_{1}(z)-\beta_{10}\left(z^{g_{01}}\right) G_{0}(z) .
$$

We have to find a rational filter $\beta_{10}(z)$ such that

$$
\left.\left(G_{1}(z) \widetilde{G}_{0}(z)\right)\right\rfloor_{g_{01}}=0 .
$$

It can be verified that the choice

$$
\beta_{01}(z)=\frac{\left(F_{1}(z) \widetilde{G}_{0}(z)\right) \downarrow_{g_{01}}}{\left(G_{0}(z) \widetilde{G}_{0}(z)\right) \downarrow_{g_{01}}}
$$

works. Clearly $G_{1}(z)$ defined in (3.5) remains rational. Now assume that we have made $G_{0}(z), G_{1}(z), \ldots, G_{s-1}(z)$ orthogonal to each other in the sense of (3.4). We wish to make $G_{s}(z)$ orthogonal to $G_{0}(z), \ldots, G_{s-1}(z)$. Define

$$
G_{s}(z)=F_{s}(z)-\sum_{i=0}^{s-1} \beta_{s i}\left(z^{g_{s i}}\right) G_{i}(z)
$$

Our aim is to choose rational filters $\beta_{s i}(z)$ appropriately. Recall that $g_{l i}$ is the gcd of $\left(n_{i}, n_{l}\right)$. With $L$ denoting the lcm of the integers $\left\{n_{i}\right\}$, we can write

$$
L=g_{s 0} c_{s 0}=g_{s 1} c_{s 1}=\cdots=g_{s, s-1} c_{s, s-1}
$$

where $c_{s i}$ are integers. Expressing $\beta_{s i}(z)$ in $c_{s i}$-fold polyphase form $\beta_{s i}(z)=\sum_{l=0}^{c_{s i}-1} z^{-l} \beta_{s i l}\left(z^{c_{s i}}\right)$, we get

$$
G_{s}(z)=F_{s}(z)-\sum_{i=0}^{s-1} \sum_{l=0}^{c_{s i}-1} \beta_{s i l}\left(z^{L}\right) z^{-l g_{s i}} G_{i}(z)
$$

We want to make $G_{s}(z)$ orthogonal to $G_{k}(z)$ for $k=$ $0,1, \ldots, s-1$. Imposing this condition and simplifying, we get [8]

$$
\begin{gathered}
\sum_{i=0}^{s-1} \sum_{i=0}^{c_{s i}-1} \beta_{s i l}(z)\left(\left(z^{m g_{s k}-l g_{s i}} G_{i}(z) \widetilde{G}_{k}(z)\right) \downarrow_{L}\right) \\
=\left(z^{m g_{s k}} F_{s}(z) \widetilde{G}_{k}(z)\right) \downarrow_{L}
\end{gathered}
$$

for $m=0,1, \ldots, c_{s k}-1$ and $k=0,1, \ldots, s-1$. So we have $\sum_{i=0}^{s-1} c_{s i}$ unknowns $\beta_{s i l}(z)$, and the same number of linear equations. The solutions $\beta_{s i}(z)$ 's are rational functions, so $G_{s}(z)$ will remain a rational transfer function. At the end of this process, we have a new set of rational transfer functions $\left\{G_{k}(z)\right\}_{k=0}^{M-1}$ satisfying (3.4). (If the determinant of the system is identically zero, we can obtain a reduced equation, and proceed to solve it [8]). The set of orthogonal functions $G_{k}(z)$ can finally be orthonormalized as described in Sec. 3.1.

\section{Stability Preservation}

It is possible that some of the filters in the orthogonalized set $G_{k}(z)$ have poles outside the unit circle. In this case, we can find rational allpass filters $\gamma_{k}(z)$ such that the new set of filters $\gamma_{k}\left(z^{n_{k}}\right) G_{k}(z)$ remain orthonormal, and are free from poles outside the unit circle [8].

How about poles on the unit circle? It can be shown that if $G_{s}(z)$ has a pole on the unit circle, then it is cancelled during the subsequent normalization step (3.1). The other possibility is that during normalization, the filter $\alpha_{k}(z)$ itself has a pole on the unit circle. It can be shown that such a pole will automatically cancel in the product (3.1). All details can be found in [8].

\section{Orthonormalization Examples}

Consider a three channel nonuniform biorthonormal filter bank with decimation ratios 4,4 and 2 . The filters that we started with were all FIR with lengths 28,28 and 10. After the orthonormalization, the resulting filters are IIR, with numerator degrees $100,28,10$ and denominator degrees $33,25,9$ respectively. These are evidently very inefficient filters, though the magnitude responses of the biorthonormal and orthonormal systems (Fig. 3.1) do not differ much! This example demonstrates that the above orthogonalization process is primarily of theoretical interest - it establishes the existence of orthonormal systems whenever biorthonormal systems exist for some $\left\{n_{i}\right\}$.

\section{TRUE DECORRELATION OF SUBBANDS}

If a uniform filter bank $\left(n_{k}=M\right)$ is such that the filters have length $\leq M$ then the system is equivalent to a transform coder. The special case of a unitary transform coder is equivalent to an orthonormal filter bank with filter length restricted to $M$. In this case, the Karhunen Loeve Transform (KLT) is known to maximize the coding

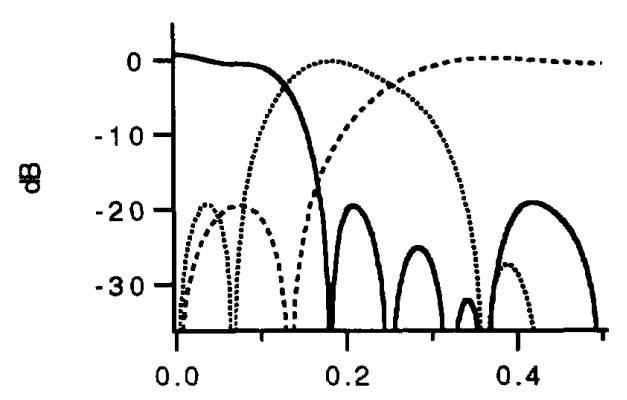

(a)

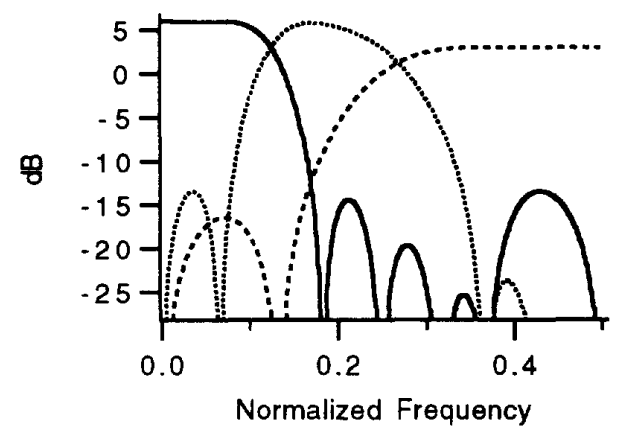

(b)

Fig.3.1. Magnitude responses of analysis filters (a) biorthonormal (b) orthonormal 
gain of the system with optimal quantizers [10], [4]. This system also decorrelates the subband signals in a limited sense. That is, at any time instant $n$, the decimated subband signals $x_{i}(n)$ and $x_{l}(n)$ are uncorrelated for $i \neq l$. However, the signals $x_{i}(n)$ and $x_{l}(m), m \neq n$ are in general not uncorrelated.

Consider now the other extreme where the subband filters are nonoverlapping ideal brickwall filters. Then the subband signals are truly decorrelated, i.e., $x_{i}(n)$ and $x_{l}(m)(i \neq l)$ are uncorrelated for any $n, m$. This leads us to ask if there is a direct connection between coding gain and subband decorrelation. Our experience shows that mere subband decorrelation has no strong connection to coding gain improvement.

To demonstrate this we develop a procedure to "truly decorrelate" subband signals using biorthonormal filter banks with rational (nonideal) filters. This is done by designing the analysis filters by taking into account the input statistics. (The KLT achieves this for subband samples at the same instant of time.) The technique is based on a modification of the orthogonalization procedure of Sec. 3.2, as applied to signals rather than filters.

We consider only the uniform $\left(n_{k}=M\right)$ case; for the nonuniform case, see [8]. Suppose we are given a wide sense stationary process $x(n)$ with rational power spectrum $S(z)$ (i.e., ratio of polynomials in $z$ ). We wish to find rational filters $H_{k}(z)$ such that the decimated subband signals are decorrelated. It turns out [8] that if we impose the orthonormality constraint on the filter bank, then this cannot be done unless $S(z)$ has the restricted form $f\left(z^{M}\right)$, where $M$ is the number of subbands. We therefore need to consider only the biorthonormal case.

\section{Uniform Biorthonormal Case}

The cross power spectrum between the subband signals $x_{i}(n)$ and $x_{k}(n)$ is

$$
S_{i k}(z)=\left(\widetilde{H}_{i}(z) H_{k}(z) S(z)\right) \downarrow_{M}
$$

We would like to force this to be zero for $i \neq k$. Given a PR (birothonormal) system with analysis filters $H_{i}(z)$, we show how to obtain a new set of analysis filters $G_{i}(z)$ such that this is true.

Set $G_{0}(z)=H_{0}(z)$. In order to make $x_{1}(n)$ and $x_{0}(n)$ decorrelated, we look for $G_{1}(z)$ in the form

$$
G_{1}(z)=H_{1}(z)-\beta_{10}\left(z^{M}\right) G_{0}(z)
$$

The decorrelation condition is

$$
\left.\left(G_{1}(z) \widetilde{G}_{0}(z) S(z)\right)\right\rfloor_{M}=0
$$

For this, we choose:

$$
\beta_{10}(z)=\frac{\left.\left(H_{1}(z) \widetilde{G}_{0}(z) S(z)\right)\right\rfloor_{M}}{\left.\left(G_{0}(z) \widetilde{G}_{0}(z) S(z)\right)\right\rfloor_{M}}
$$

We can continue in this manner by modifying the results of Sec. 3.2. For example in the sth step we have $G_{s}(z)$ $=H_{s}(z)-\sum_{k=0}^{s-1} \beta_{s k}\left(z^{M}\right) G_{k}(z)$, and $\beta_{s k}(z)$ can be chosen to satisfy the decorrelation condition. The synthesis filters for biorthonormality can then be found by inverting the polyphase matrix; see [8] for details.

\section{Example of Subband Decorrelation}

We consider a lowpass AR(6) process [10], and a two channel filter bank, and initialize the filter bank coefficients with a paraunitary system with FIR filters of order 7 (filter $8 \mathrm{~A}$ in [11]). The decorrelating filters do not form a paraunitary system. The analysis filters are FIR, with order 7 and 18 respectively, and the synthesis filters are IIR of orders 18 and 12 .

Coding gain. The coding gain [10], [4] of the initial paraunitary system is $G_{P U}=3.09$. After subband decorrelation it drops to $G_{D C}=2.8$. So decorrelation of the subband signals itself does not necessarily increase the coding gain! On the other hand, the coding gain of the ideal brick wall two channel filter bank is $G_{S B C}=3.38$ and the subband signals are truly decorrelated.

Comments. Filters that decorrelate the subband signals (hence the coding gain achieved with them) depend heavily on the filters that we start with. Since the filter banks that decorrelate the subband signals are not paraunitary, there is no theoretical guarantee that the coding gain will be greater than one!

\section{References}

[1] Vaidyanathan, P. P., "Orthonormal and biorthonormal filter banks as convolvers, and convolutional coding gain", IEEE Trans. SP, June 1993.

[2] Vetterli, M., and Herley, C. "Wavelets and filter banks," IEEE Trans. SP, vol. SP-40, Sept. 1992.

[3] A. K. Soman and P. P. Vaidyanathan, "On Orthonormal Wavelets and Paraunitary Filter Banks", IEEE Trans. SP, March 1993.

[4] P. P. Vaidyanathan, Multirate systems and filter banks, Prentice Hall, 1993.

[5] P. Q. Hoang and P. P. Vaidyanathan, "Non-Uniform Multirate Filter Banks: Theory and Design", Proc. IEEE ISCAS, pp. 371-374, Portland, May 1989.

[6] J. Kovačević and M. Vetterli, "Perfect Reconstruction Filter Banks with Rational Sampling Rate Changes", Proc. IEEE ICASSP, pp. 1785-1788, Canada, May 1991. [7] K. Nayebi, T. P. Barnwell, and M. Smith, "The design of perfect reconstruction nonuniform band filter banks," Proc. IEEE ICASSP, pp. 1781-1784, Canada, May 1991.

[8] I. Djokovic and P. P. Vaidyanathan, "Biorthonormal filter banks: properties and orthonormalization procedures," Tech. rep., Caltech, Jan. 1993.

[9] R. M. Young, "An Introduction to Nonharmonic Fourier Series", Academic Press, New York, 1980.

[10] N. S. Jayant and P. Noll, "Digital Coding of Waveforms: Principles and Applications to Speech and Video", Prentice-Hall, 1984.

[11] P. P. Vaidyanathan and P. Q. Hoang, "Lattice Structures for Optimal Design and Robust Implementation of Two Channel Perfect Reconstruction QMF Banks", IEEE Trans. ASSP pp. 81-94, Jan. 1988. 